\title{
Engineering of Magnetic Properties of Magnetic Microwires
}

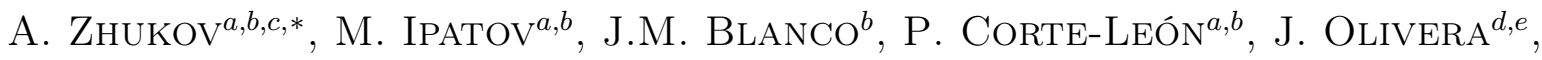 \\ J. Gonzalez ${ }^{a}$ And V. ZhuKova ${ }^{a, b}$ \\ ${ }^{a}$ Dpto. de Física de Matereriales, Univesity of Basque Country, 20018, San Sebastián, Spain \\ ${ }^{b}$ Dpto. de Física Aplicada, EUPDS, Univesity of Basque Country, 20018, San Sebastian, Spain \\ ${ }^{c}$ IKERBASQUE, Basque Foundation for Science, 48011, Bilbao, Spain \\ ${ }^{d}$ Laboratorio de la Dirección General de Aduanas, Santo Domingo, República Dominicana \\ ${ }^{e}$ Instituto Tecnológico de Santo Domingo (INTEC), Santo Domingo, República Dominicana
}

\begin{abstract}
We present an overview of the factors affecting soft magnetic properties, fast domain wall propagation and giant magnetoimpedance (GMI) effect in thin amorphous wires. The magnetoelastic anisotropy is one of the most important parameters that determine the magnetic properties of glass-coated microwires and therefore annealing can be very effective for manipulation the magnetic properties of amorphous ferromagnetic glass-coated microwires. Increasing of DW velocity in Fe-rich and Fe-Ni based (low Ni content) microwires is achieved after annealing. After heat treatment of Co-rich microwires we can observe transformation of inclined hysteresis loops to rectangular and coexistence of fast magnetization switching and GMI effect in the same sample. On the other hand stress annealing of Fe- and Co-rich microwires allows achievement of considerable magnetic softening and GMI effect enhancement.
\end{abstract}

DOI: 10.12693/APhysPolA.133.321

PACS/topics: 75.60.Ej, 75.60.Jk

\section{Introduction}

Novel soft magnetic wires with reduced dimensions and improved magnetic and magneto-transport properties are quite demanded by developing industries [1,2]. Studies of crystalline magnetic wires exhibiting either giant magnetoimpedance (GMI) or single domain wall (DW) propagation attracted attention along many years $[3,4]$. It is worth mentioning that magnetic properties of crystalline materials are affected by defects of crystalline structure, grain size, crystalline texture, grain boundaries, etc. [5]. Therefore amorphous wires produced using melt quenching characterized by the absence of longrange atomic order have attracted considerable attention since 80-th [5-7].

One of the principal advantages of amorphous magnetic wires is that magnetic softness can be achieved even in as-prepared samples avoiding in this way complex and durable post-processing. Moreover the technology of melt quenching (also known as rapid quenching) is not expensive and rather fast $[1,2,5-7]$. Cylindrical symmetry of melt quenched magnetic wires allows considerable enhancement of GMI effect and single DW velocity as compared to other families of soft magnetic materials [8].

The GMI effect is satisfactory interpreted in terms of classical electrodynamics considering skin effect in magnetically soft conductor [8-10]. High circumferential magnetic permeability typical for amorphous magnetic wires is the necessary feature for observation of high GMI effect $[6-8]$.

\footnotetext{
*corresponding author; e-mail: arkadi.joukov@ehu.es
}

Similarly to giant magneto-resistance (GMR) effect the GMI effect is typically expressed by the magnetoimpedance ratio, $\Delta Z / Z$, defined as:

$$
\Delta Z / Z=\left[Z(H)-Z\left(H_{\max }\right)\right] / Z\left(H_{\max }\right),
$$

where $H_{\max }$ is the maximum axial DC magnetic field (usually up to few $\mathrm{kA} / \mathrm{m}$ ).

The main interest in GMI effect is related to one of the largest magnetic field sensitivities (up to $10 \% / \mathrm{A} / \mathrm{m}$ ) among non-cryogenic effects making it quite interesting for application in magnetic sensors and magnetometers [9-12].

The commonly used technologies for creation of magnetic sensors and magnetometers are Hall-effect, GMR and fluxgates. GMI technology allows achievement of nearly $1 \mathrm{pT}$ magnetic field sensitivity making these sensors suitable for the detection of biomagnetic fields [13].

For extended applications of GMI sensors not only high magnetic field sensitivity, but also micro-size and low cost are quite relevant parameters [8]. Therefore development of thin magnetic wires with enhanced magnetic softness is highly demanded by industries.

Actually there are a few families of amorphous magnetic wires that can be prepared using different fabrication processes involving melt quenching:

1. Conventional amorphous wires prepared using "inrotating water" method (diameters of the order of $100 \mu \mathrm{m})[6,7,9]$. The disadvantage of these wires is rather thick diameter. Therefore cold-drawn method is proposed for the diameter reduction [14].

2. Melt extracted amorphous microwires (diameters of 30-60 $\mu \mathrm{m})$ known since beginning of 90-th $[15,16]$. These microwires present not perfectly cylindrical shape that can affect the magnetic properties and hence GMI effect. 
3. Glass-coated (composite) microwires (metallic nucleus diameters of $0.5-40 \mu \mathrm{m}$ ) prepared using socalled modified Taylor-Ulitovsky (also known as quenching-and-drawing method) known since 60th [17], but extensively studied in 90-th [18-19]. The fabrication method involves rapid solidification from the melt of both metallic alloys in form of wire and glass-coating. The characteristic feature of these microwires is the enhanced magnetoelastic anisotropy arising by rapid quenching itself as well by the difference in thermal expansion coefficients $[5,18,19]$.

The other quite interesting feature of different families of magnetic wires is the possibility to observe single domain wall (DW) propagation [18, 20, 21]. From the viewpoint of applications the speed of single DW propagation is essentially relevant. In the case of cylindrical amorphous microwires exhibiting spontaneous magnetic bistability the DW speed above $1 \mathrm{~km} / \mathrm{s}$ can be easily achieved [18, 22, 23]. Aforementioned magnetic bistability is characterized by squired hysteresis loops related to a large and single Barkhausen jump within single domain axially magnetized inner core of wire $[18,22,23]$.

Consequently studies of single DW propagation in amorphous glass-coated microwires are quite important for understanding of the ways to enhance the DW velocity in other materials.

Aforementioned glass-coated microwires present a number of advantages, such as:

- fabrication of continuous long pieces of microwire up to $10000 \mathrm{~m}$;

- control and adjustment of geometrical parameters (inner core diameter and glass thickness) during the fabrication process $[1,8]$;

- existence of insulating biocompatible glass-coating suitable for various applications including biological and medical applications [24];

- control of internal stresses and therefore magnetoelastic anisotropy through the ratio, $\rho$, between metallic nucleus diameter, $d$, and total microwire diameter, $D(\rho=d / D)[1,25,26]$;

- thinnest metallic nucleus diameter among the magnetic wires prepared using casting methods families [27, 28];

- enhanced corrosion resistivity and mechanical properties due to glass coating.

In spite of aforementioned advantages this method meets some complexities related with peculiarities of the glasscoated preparation technique $[1,5]$. Moreover recently formation of the interface layer between the metallic nucleus and glass-coating with typical thickness of about $0.5 \mu \mathrm{m}$ is reported [29].
All these peculiarities affect magnetic and structural properties of cast microwires. In spite of these disadvantages the highest GMI ratios (up to 600\%) are reported for Co-rich glass-coated microwires [30, 31]. Therefore we focused our research on engineering of magnetic softness, GMI effect and single DW velocity in amorphous and nanocrystalline glass-coated microwires.

\section{Experimental method}

We prepared Fe-Co-Ni based glass-coated microwires with either positive or negative magnetostriction coefficient, $\lambda_{s}$, and with different $\rho$-ratios $[10]$.

Magnetic field dependencies of impedance, $Z$, and GMI ratio, $\Delta Z / Z$ (defined by Eq. (1)), were obtained. We used specially designed micro-strip sample holder placed inside a sufficiently long solenoid allowing creating a homogeneous magnetic field, $H$. The sample impedance, $Z$, was measured using vector network analyzer from the reflection coefficient $S_{11}$ as described elsewhere [32]. The off-diagonal GMI component, $Z_{z \varphi}$, has been evaluated through the transmission coefficient $S_{21}$ [32]. Employed method allowed extending of the frequency range up to GHz-range [32].

We measured the DW velocity using modified SixtusTonks-like experiments described elsewhere [18, 22, 23]. 3 pick-up coils, mounted along the microwire and placed inside the long solenoid allowed to measure the velocity of propagating DW $[18,22,23]$. The electromotive force (emf) sharp peaks from the 3 pick-up coils detected by the digital oscilloscope upon passing the propagating wall allowed estimation of the DW velocity as:

$$
v=\frac{l}{\Delta t}
$$

where $l$ is the distance between a pair of pick-up coils and $\Delta t$ is the time difference between the maximum in the induced emf.

Hysteresis loops have been measured using vibrating sample magnetometer VSM and fluxmetric technique described elsewhere [18, 23].

The magnetostriction coefficient, $\lambda_{s}$ of the samples has been evaluated using small angle magnetization rotation (SAMR) method initially proposed for the materials exhibiting only magnetization rotation [32] and recently extended for the case of magnetic microwires [33].

We evaluated magnetic properties of as-prepared and annealed microwires. Annealing has been performed in a conventional furnace.

\section{Experimental results and discussion}

\subsection{Engineering of magnetic properties in as-prepared magnetic microwires}

As can be appreciated from Fig. 1, better magnetic softness in as-prepared microwires is observed for CoFe-rich microwires presenting vanishing magnetostriction coefficients. The magnetostriction coefficient, $\lambda_{s}$, in the 
classical system $\left(\mathrm{Co}_{x} \mathrm{Fe}_{1-x}\right){ }_{75} \mathrm{Si}_{15} \mathrm{~B}_{10}$ can be tuned by the chemical composition: i.e. from $\lambda_{s} \approx-5 \times 10^{-6}$ at $x=1$, to $\lambda_{s} \approx 38 \times 10^{-6}$ at $x \approx 0.2$. Nearly-zero $\lambda_{s}$-values can be achieved at $\mathrm{Co} / \mathrm{Fe} \approx 70 / 5[34,35]$.

Consequently, considering magnetic properties of asprepared microwires they can be classified into three main groups:

- Fe-based wires with positive (of the order of $10^{-5}$ ) $\lambda_{s}$-values presenting spontaneous magnetic bistability (rectangular hysteresis loop, see Fig. 1a);

- Co-based wires with negative (of the order of $10^{-6}$ ) $\lambda_{s}$-values exhibiting almost non-hysteretic linear magnetization curves with rather high magnetic anisotropy field (see Fig. 1c);

- Co-based wires with vanishing $\lambda_{s}$-values presenting best magnetic softness (see Fig. 1b).

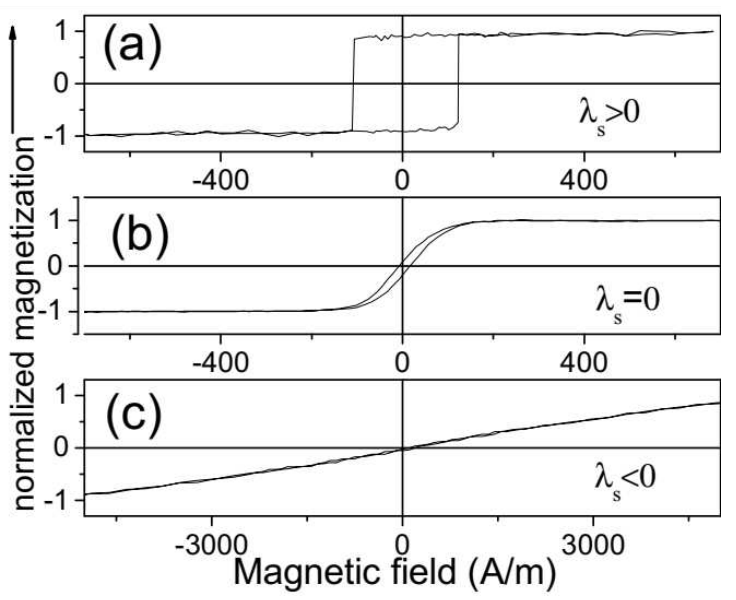

Fig. 1. Effect of magnetostriction on hysteresis loops of glass coated microwires: (a) Fe-rich $\left(\mathrm{Fe}_{70} \mathrm{~B}_{15} \mathrm{Si}_{10} \mathrm{C}_{5}\right)$,

(b) Fe-Co-rich $\left(\mathrm{Co}_{67.1} \mathrm{Fe}_{3.8} \mathrm{Ni}_{1.4} \mathrm{Si}_{14.5} \mathrm{~B}_{11.5} \mathrm{Mo}_{1.7}\right)$ and

(c) Co-rich $\left(\mathrm{Co}_{77.5} \mathrm{Si}_{15} \mathrm{~B}_{7.5}\right)$.

The most appreciable difference from the other families of magnetic wires is observed in Co-rich microwires with negative (of the order of $10^{-6}$ ) $\lambda_{s}$-values: they present almost non-hysteretic magnetization curves, while Co-rich conventional wires exhibit rectangular hysteresis loop.

Strength of internal stresses arising during the fabrication process is the other important factor affecting magnetic properties of glass-coated microwires.

As mentioned above, the strength of internal stresses in glass-coated microwires depends on a few parameters. Basically there are three main factors affecting total internal stresses: i) quenching stresses associated to the rapid quenching from the melt of the metallic ingot; ii) stresses related to the different thermal expansion coefficients of metallic alloy and glass solidifying together and iii) stresses associated to the drawing of solidifying wire $[19,25,26]$. Additionally the strength of the internal stresses, $\sigma_{i}$, is directly to the $\rho$-ratio: $\sigma_{i}$ increase with decreasing of the $\rho$-ratio $[25,26,26]$. Therefore the internal stresses can be controlled though the fabrication parameters through the $\rho$-ratio.

As an example, in Co-rich microwires of the same chemical composition (with low and negative $\lambda_{s}$-values of about $10^{-7}$ ) presenting linear hysteresis loops the field of magnetic anisotropy decrease with $\rho$-ratio increasing (Fig. 2a). In the case of Fe-rich microwires (with positive $\lambda_{s}$-values of about $35 \times 10^{-6}$ ) the coercivity, $H_{c}$, decreases with $-\rho$-ratio increasing (Fig. $2 \mathrm{~b}$ ).
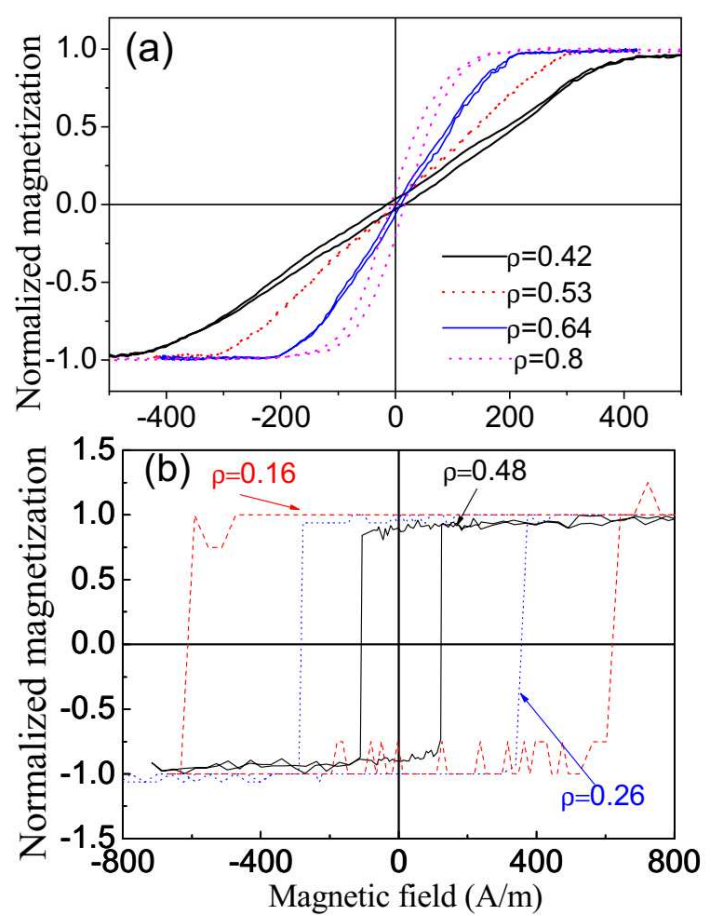

Fig. 2. Effect of $\rho$-ratio on hysteresis loops of $\mathrm{Co}_{67.1} \mathrm{Fe}_{3.8} \mathrm{Ni}_{1.4} \mathrm{Si}_{14.5} \mathrm{~B}_{11.5} \mathrm{Mo}_{1.7}$ microwires (a) and of $\mathrm{Fe}_{70} \mathrm{~B}_{15} \mathrm{Si}_{10} \mathrm{C}_{5}$ amorphous microwires (b).

Both aforementioned parameters affect the magnetoelastic anisotropy, $K_{m e}$, given as:

$$
K_{m e}=3 / 2 \lambda_{S} \sigma_{i},
$$

where $\lambda_{S}$ is the saturation magnetostriction and $\sigma_{i}$ is the internal stress.

Consequently the magnetoelastic anisotropy is one of the most relevant parameters affecting magnetic properties of glass-coated microwires.

It is theoretically predicted and experimentally confirmed $[37,38]$ that the GMI ratio and magnetic field dependence are determined by the magnetic anisotropy. Therefore the difference in $\Delta Z / Z /(H)$ dependencies experimentally found in Fe-rich and Co-rich microwires (see Fig. 3) is not surprising: a decay of impedance, $Z$, versus $H$ is observed in $\mathrm{Fe}_{75} \mathrm{~B}_{9} \mathrm{Si}_{12} \mathrm{C}_{4}$ microwires, while one order higher maximum GMI ratio, $\Delta Z / Z_{m}$, and double - peak $\Delta Z / Z(H)$ dependencies are experimentally observed for $\mathrm{Co}_{69.2} \mathrm{Fe}_{4.1} \mathrm{~B}_{11.8} \mathrm{Si}_{13.8} \mathrm{C}_{1.1}$ magnetic microwires (Fig. 3). 


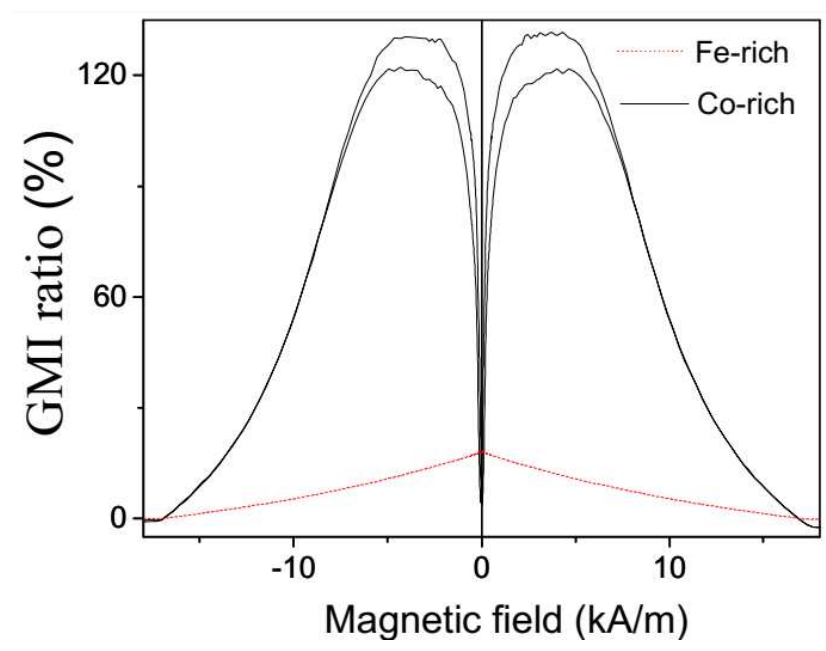

Fig. 3. $\Delta Z / Z(H)$ dependencies of $\mathrm{Fe}_{75} \mathrm{~B}_{9} \mathrm{Si}_{12} \mathrm{C}_{4}$ and $\mathrm{Co}_{69.2} \mathrm{Fe}_{4.1} \mathrm{~B}_{11.8} \mathrm{Si}_{13.8} \mathrm{C}_{1.1}$ microwires measured at $500 \mathrm{MHz}$.

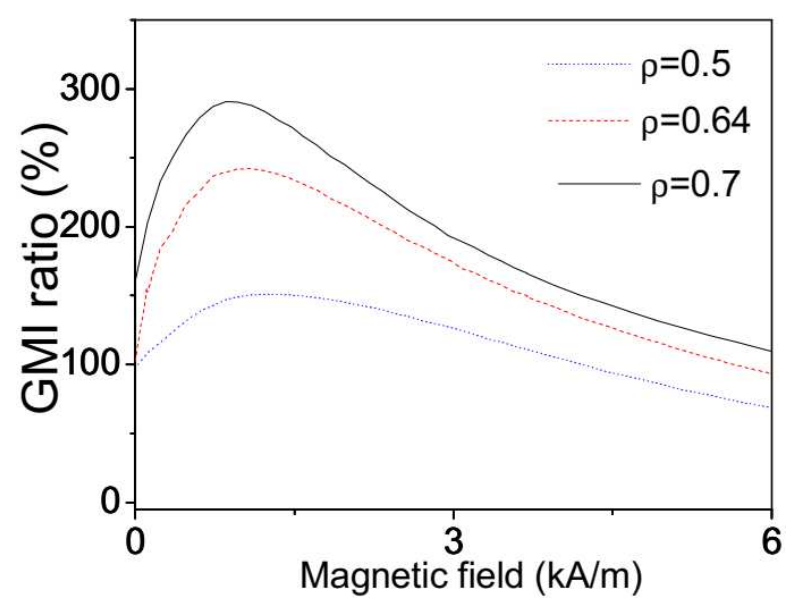

Fig. 4. $\Delta Z / Z(H)$ dependencies of as-prepared $\mathrm{Co}_{67} \mathrm{Fe}_{3.85} \mathrm{Ni}_{1.45} \mathrm{~B}_{11.5} \mathrm{Si}_{14.5} \mathrm{Mo}_{1.7}$ microwires with different $\rho$-ratios measured at $500 \mathrm{MHz}$.

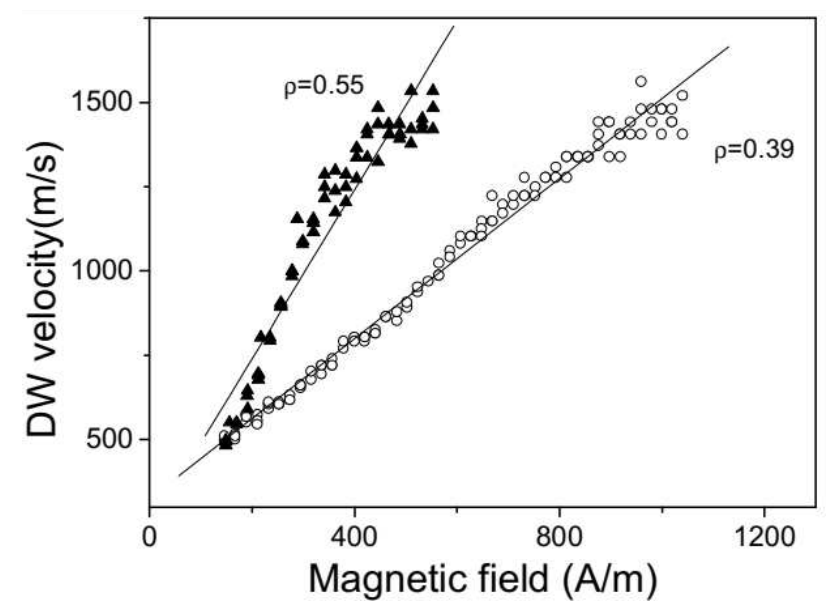

Fig. 5. $\quad v(H)$ dependencies for $\mathrm{Co}_{41.7} \mathrm{Fe}_{36.4} \mathrm{Si}_{10.1} \mathrm{~B}_{11.8}$ microwires with different ratios $\rho$. Solid lines show a linear fit of experimental data.
The influence of internal stresses on GMI effect is evidenced by the $\Delta Z / Z /(H)$ dependencies measured in Corich microwires with the same composition of the metallic nucleus but with different $\rho$-ratios: both $\Delta Z / Z_{m}$ and field of $\Delta Z / Z /(H)$ maximum, $H_{m}$, are affected by $\rho$-ratio for $\mathrm{Co}_{67.1} \mathrm{Fe}_{3.8} \mathrm{Ni}_{1.4} \mathrm{Si}_{1.45} \mathrm{~B}_{11.5}$ microwires with different $\rho$-ratio (see Fig. 4).

Not only GMI effect, but also other magnetic properties, such as DW dynamics are affected by the magnetoelastic anisotropy.

The effect of internal stresses (modified through the $\rho$-ratio) on $v(H)$ dependence of microwires of the same $\left(\mathrm{Co}_{41.7} \mathrm{Fe}_{36.4} \mathrm{Si}_{10.1} \mathrm{~B}_{11.8}\right)$ composition is shown in Fig. 5 . In according to our measurements these microwires present magnetostriction coefficient, $\lambda_{s} \approx 20 \times 10^{-6}$, that is in good agreement to earlier published results [39].

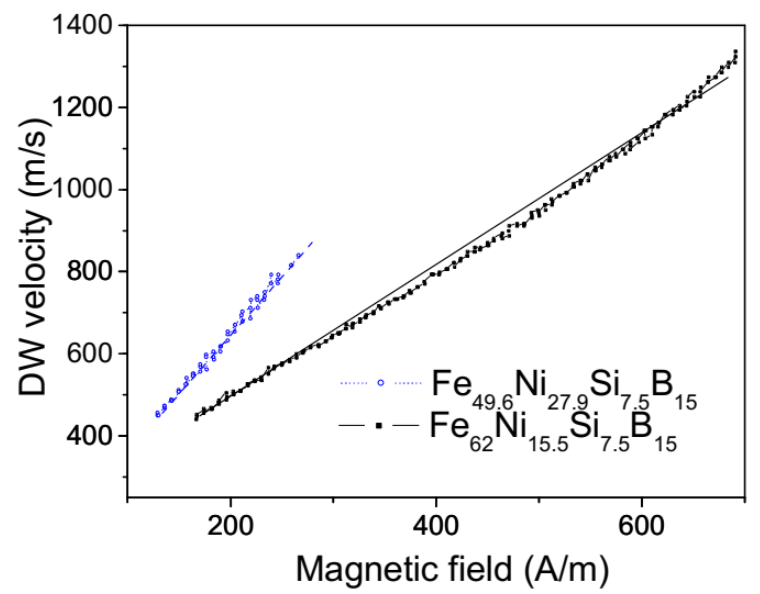

Fig. 6. $v(H)$ dependencies for $\mathrm{Fe}_{49.6} \mathrm{Ni}_{27.9} \mathrm{Si}_{7.5} \mathrm{~B}_{15}$ $(\rho \approx 0.43)$ and $\mathrm{Fe}_{62} \mathrm{Ni}_{15.5} \mathrm{Si}_{7.5} \mathrm{~B}_{15}(\rho \approx 0.42)$ microwires with different magnetostriction coefficients. Solid lines show a linear fit of experimental data.

Similar dependence has been observed in Fe-Ni- based microwires $\left(\mathrm{Fe}_{62} \mathrm{Ni}_{15.5} \mathrm{Si}_{7.5} \mathrm{~B}_{15}\right.$ and $\mathrm{Fe}_{49.6} \mathrm{Ni}_{27.9} \mathrm{Si}_{7.5} \mathrm{~B}_{15}$ ) with quite similar $\rho$-ratios $(\rho \approx 0.43$ and 0.42 respectively) but with different $\lambda_{s}$-values $\left(\lambda_{s} \approx 25 \times 10^{-6}\right.$ and $17 \times 10^{-6}$ respectively): decreasing of DW velocity with increase of magnetoelastic anisotropy (see Fig. 6).

Consequently, magnetic properties and the GMI ratio of as-prepared microwires can be easily tailored by the magnetoelastic anisotropy though changing of $\rho$-ratio or $\lambda_{s}$-values.

\subsection{Tuning of magnetic properties} in magnetic microwires by thermal treatment

Considering high internal stresses arising during the preparation of composite microwires we considered to tailor the magnetoelastic anisotropy as well as magnetic properties by thermal treatment.

Surprisingly considerable magnetic hardening is observed in almost all Co-rich glass-coated microwires after annealing $[40,41]$. Considerable coercivity growth observed in $\mathrm{Co}_{69.2} \mathrm{Fe}_{4.1} \mathrm{~B}_{11.8} \mathrm{Si}_{13.8} \mathrm{C}_{1.1}$ and 


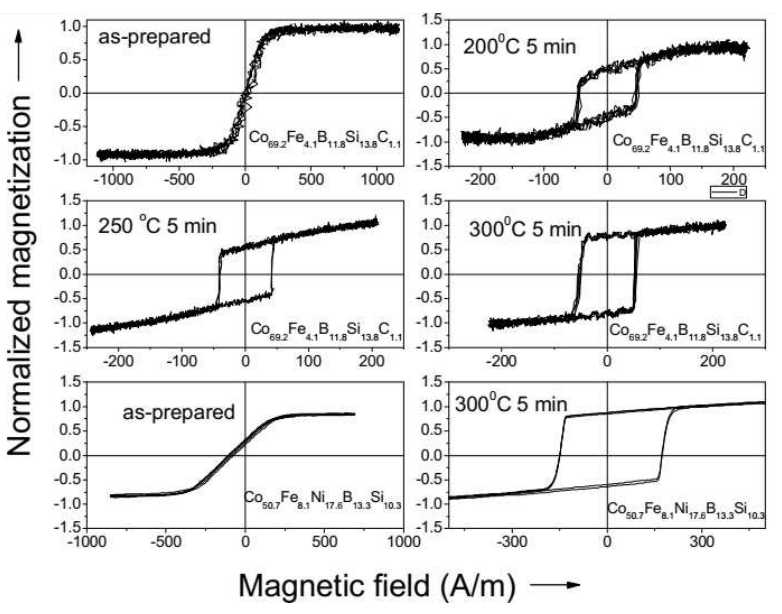

Fig. 7. Hysteresis loops of as-prepared and annealed for $5 \mathrm{~min}$ at different temperatures $\mathrm{Co}_{69.2} \mathrm{Fe}_{4.1} \mathrm{~B}_{11.8} \mathrm{Si}_{13.8} \mathrm{C}_{1.1} \quad$ and $\mathrm{Co}_{50.7} \mathrm{Fe}_{8.1} \mathrm{Ni}_{17.6} \mathrm{~B}_{13.3} \mathrm{Si}_{10.3}$ microwires.

$\mathrm{Co}_{50.7} \mathrm{Fe}_{8.1} \mathrm{Ni}_{17.6} \mathrm{~B}_{13.3} \mathrm{Si}_{10.3}$ microwires after annealing can be appreciated (Fig. 7).

For interpretation of unexpected magnetic hardening of Co-rich microwires after annealing we must consider the internal stress relaxation as well as related changes of domain structure: growing of the inner axially magnetized core induced by stress relaxation [40-42]. Indeed in most of the volume of metallic nucleus the axial stresses induced by preparation method are the dominant one $[25,36]$. In negative magnetostrictive microwires these internal stresses result in circumferential easy magnetization direction. Consequently stress relaxation dives rise to inner axially magnetized core volume increasing.

Aforementioned changes of domain structure and hence of hysteresis loops are reflected in change of the GMI effect after annealing of Co-rich microwires (Fig. 8a): maximum GMI ratio, $\Delta Z / Z_{m}$, considerably decrease after annealing. On the other hand stress annealing of the same microwires results in growing of GMI ratio (Fig. 8b).

This opposite tendency in evolution of GMI ratio after annealing and stress-annealing must be related to stressinduced anisotropy. It is well-known that induced magnetic anisotropy can play an important role in annealed amorphous materials (especially in the presence of magnetic field or/and applied stress) [43].

The origin of induced anisotropy in amorphous materials is commonly associated to either pair ordering or back stresses $[1,39,40,44-46]$. Consequently remarkable changes of magnetic properties are expected for amorphous materials containing more than one transition metal. In the present case glass-coated microwires annealed under stress and without stress at the same annealing conditions present considerably different hysteresis loops: stress annealed samples present lower coercivity (Fig. 9).

Even stronger hysteresis loops changes are observed after stress-annealing of Fe-rich samples: a considerable
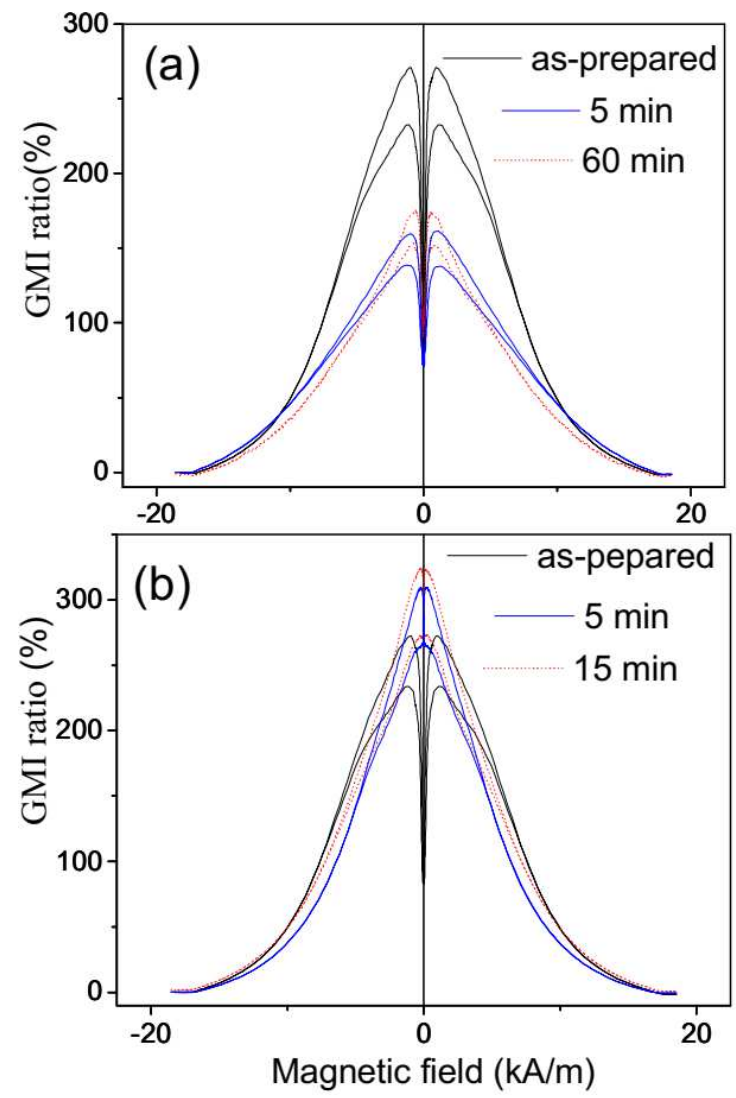

Fig. 8. $\Delta Z / Z(H) \quad$ dependencies of $\mathrm{Co}_{69.2} \mathrm{Fe}_{4.1} \mathrm{~B}_{11.8} \mathrm{Si}_{13.8} \mathrm{C}_{1.1}$ microwires: (a) as-prepared and annealed at $T_{a n n}=300{ }^{\circ} \mathrm{C}$ for $t_{a n n}=5 \mathrm{~min}$ and $t_{a n n}=60 \mathrm{~min}$ and (b) as-prepared and stress- annealed at $T_{a n n}=300^{\circ} \mathrm{C}$ for $t_{a n n}=5 \mathrm{~min}$ and $t_{a n n}=15 \mathrm{~min}$ (b). All measurements are performed at $200 \mathrm{MHz}$.

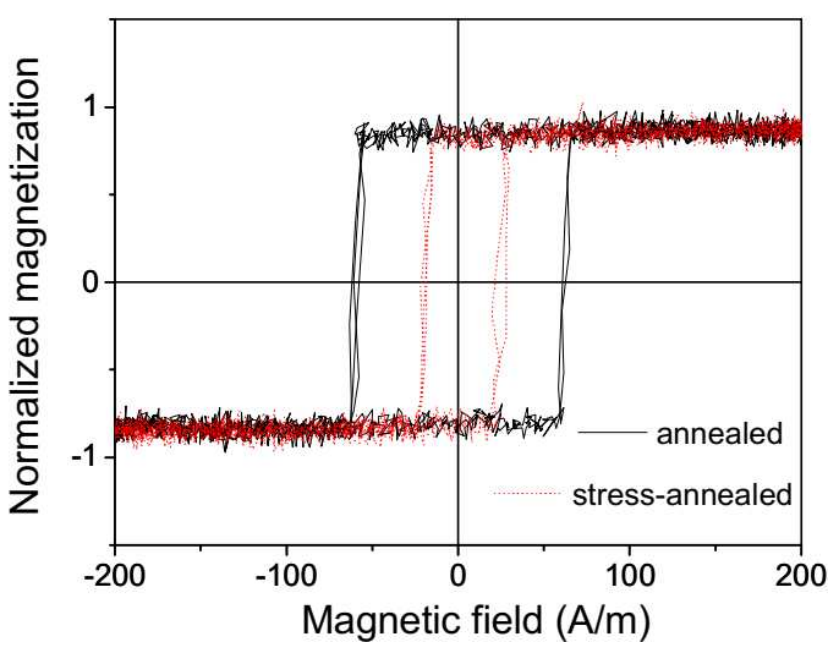

Fig. 9. Hysteresis loops of annealed and stressannealed $\mathrm{Co}_{69.2} \mathrm{Fe}_{4.1} \mathrm{~B}_{11.8} \mathrm{Si}_{13.8} \mathrm{C}_{1.1}$ microwires at $T_{a n n}=300^{\circ} \mathrm{C}$ for $t_{a n n}=45 \mathrm{~min}$. 

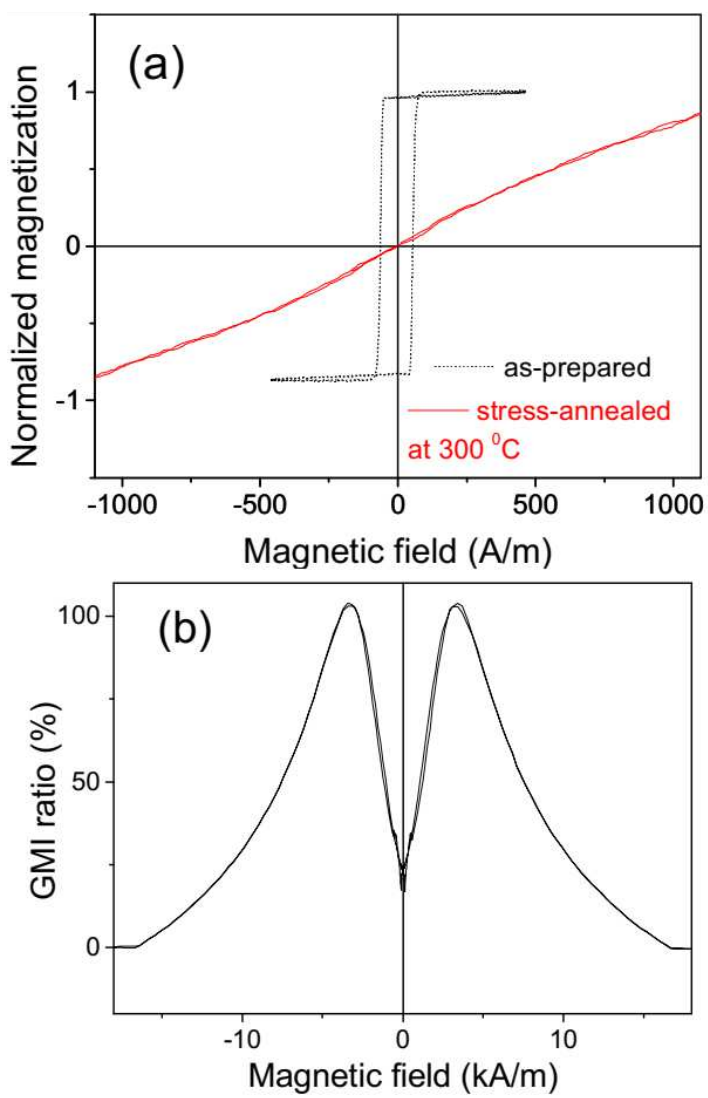

Fig. 10. Hysteresis loops as-prepared and stress annealed at $T_{a n n}=300{ }^{\circ} \mathrm{C}$ for $t_{a n n}=60 \mathrm{~min}$ (a) and $\Delta Z / Z(H)$ dependence of stress-annealed $\mathrm{Fe}_{75} \mathrm{~B}_{9} \mathrm{Si}_{12} \mathrm{C}_{4}$ microwires measured at $500 \mathrm{MHz}$ (b).

magnetic softening and coercivity reduction is observed in stress annealed $\mathrm{Fe}_{75} \mathrm{~B}_{9} \mathrm{Si}_{12} \mathrm{C}_{4}$ microwires (Fig. 10).

A considerable magnetic softening and changing of the hysteresis loop character observed after stress-annealing giving rise to increasing of GMI ratio and change of $\Delta Z / Z(H)$ dependence. Stress annealed $\mathrm{Fe}_{75} \mathrm{~B}_{9} \mathrm{Si}_{12} \mathrm{C}_{4}$ microwires present double-peak $\Delta Z / Z(H)$ dependence typical for magnetic wires with transverse magnetic anisotropy.

Stress relaxation after annealing affects not only hysteresis loops and GMI but also the DW dynamics of microwires: considerable DW velocity enhancement can be observed in Fe and Fe-Ni based microwires after annealing (Fig.11a). Additionally single DW propagation can be also observed in Co-rich microwires in which magnetic bistability is induced by annealing (Fig.11b). The same $\mathrm{Co}_{69.2} \mathrm{Fe}_{4.1} \mathrm{~B}_{11.8} \mathrm{Si}_{13.8} \mathrm{C}_{1.1}$ microwire presents also rather high (above 150\%) GMI ratio (as shown in Fig. 8).

We must underline that the properties presented by annealed Co-rich microwires are unique. They present both most interesting features in the same sample: fast DW propagation (up to $2.5 \mathrm{~km} / \mathrm{s}$ ) and high GMI effect $(\Delta Z / Z \approx 150 \%)$. Aforementioned combination of magnetic properties of Co-rich microwire with magnetic bistability induced by annealing must be attributed to
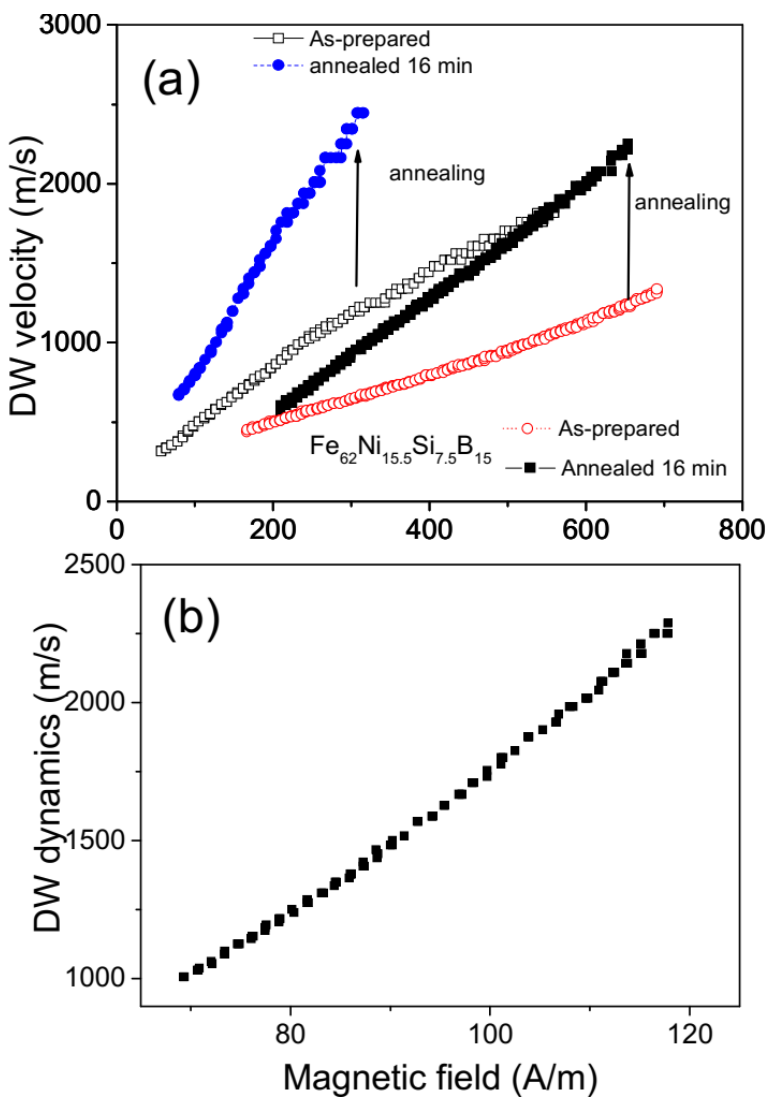

Fig. 11. Dependencies of DW velocity as a function of magnetic field, $H$ measured in as-prepared and annealed for $16 \mathrm{~min}$ at $T_{a n n} \approx 410{ }^{\circ} \mathrm{C} \mathrm{Fe}_{75} \mathrm{~B}_{9} \mathrm{Si}_{12} \mathrm{C}_{4}$ and $\mathrm{Fe}_{62} \mathrm{Ni}_{15.5} \mathrm{Si}_{7.5} \mathrm{~B}_{15}$ (a) and annealed at $T_{a n n} \approx 30{ }^{\circ} \mathrm{C}$ for 5 min $\mathrm{Co}_{69.2} \mathrm{Fe}_{4.1} \mathrm{~B}_{11.8} \mathrm{Si}_{13.8} \mathrm{C}_{1.1}$ (b) microwires.

peculiar domain structure. As mentioned above, magnetic bistability typically observed in Fe-rich microwires is explained considering existence of inner axially magnetized singe domain [47]. Therefore for Co-rich microwires with induced magnetic bistability we must consider the domain structure consisting of single-domain inner axially magnetized core and outer domain shell with high circumferential magnetic permeability [48]. This assumption is supported by quite high GMI ratio of annealed Corich microwires exhibiting rectangular hysteresis loop.

\section{Conclusions}

Thin magnetic wires present a number of magnetic properties suitable for various technological applications: excellent magnetic softness, giant magnetoimpedance (GMI) effect and fast domain wall propagation. Low coercivity and high GMI effect have been observed in asprepared Co-rich microwires with negative magnetostriction coefficient. Rectangular hysteresis loops and fast domain wall propagation are observed in as-prepared Fe-rich microwires with positive magnetostriction coefficient. Annealing allows considerable modification of hysteresis loops, magnetic softness, domain wall dynamic and GMI effect. Increasing of DW velocity in Fe-rich 
and $\mathrm{Fe}-\mathrm{Ni}$ based (low $\mathrm{Ni}$ content) microwires is achieved by annealing. After annealing of Co-rich microwires we can observe transformation of inclined hysteresis loops to rectangular and coexistence of fast magnetization switching and GMI effect in the same sample. On the other hand stress annealing of Fe- and Co-rich microwires allows achievement of considerable magnetic softening and GMI effect enhancement.

\section{Acknowledgement}

This work was supported by Spanish MINECO under MAT2013-47231-C2-1-P and by the Government of the Basque Country under the scheme of "Ayuda a Grupos Consolidados" (Ref.: IT954-16) and Elkartek (RTM 4.0) projects. Technical and human support provided by SGIker (UPV/EHU,) is gratefully acknowledged. VZ and $\mathrm{AZ}$ wish to acknowledge the support of the Basque Government under Program of Mobility of the Researchers of the Basque Government (grants MV-2017-1-0030 and MV-2017-1-0025 respectively).

\section{References}

[1] High Performance Soft Magnetic Materials, Ed. A. Zhukov), Springer Series in Materials Science, vol 252, Springer International Publishing, 2017, p. 216.

[2] D.C. Jiles, Acta Mater. 51, 5907 (2003).

[3] K.J. Sixtus, L. Tonks, Phys.Rev. 42, 419 (1932).

[4] E.P. Harrison, G. L. Turney, H. Rowe, Nature 135, 961 (1935).

[5] A. Zhukov, M. Ipatov, A. Talaat, J.M. Blanco, B. Hernando, L. Gonzalez-Legarreta, J.J. Suńol, V Zhukova, Crystals 7, 41 (2017).

[6] I. Ogasawara, S. Ueno, IEEE Trans. Magn. 31, 1219 (1995).

[7] L.V. Panina, K. Mohri, Appl Phys Lett. 65, 1189 (1994).

[8] R. Beach, A. Berkowitz, Appl. Phys. Lett. 64, 3652 (1994).

[9] K. Mohri, T. Uchiyama, L.P. Shen, C.M. Cai, L.V. Panina, J. Magn. Magn. Mater. 249, 351 (2002).

[10] Y. Honkura, J. Magn. Magn. Mater. 249, 375 (2002).

[11] L. Ding, S. Saez, C. Dolabdjian, L.G.C. Melo, A. Yelon, D. Ménard, IEEE Sensors J. 9, 159 (2009).

[12] S. Gudoshnikov, N. Usov, A.Nozdrin, M. Ipatov, A. Zhukov, V. Zhukova, Phys. Stat. Sol. (a) 211980 (2014).

[13] T. Uchiyama, K. Mohri, Sh. Nakayama, IEEE Trans. Magn. 47, 3070 (2011).

[14] I. Ogasawara, K Mohri, IEEE Trans. Magn. 26, 1795 (1990).

[15] P. Rudkowski, G. Rudkowska, J.O. Strom-Olsen, Mater. Sci. Eng. A 133, 158 (1991).

[16] V. Zhukova, A. Zhukov, V. Kraposhin, A. Prokoshin, J. Gonzalez, Sens. Actuators (A) 106, 225 (2003).

[17] R. Gemperle, L. Kraus, J. Schneider, Czech J. Phys. B 28, 1138 (1978)
[18] V. Zhukova, J. M. Blanco, M. Ipatov, A. Zhukov, J. Appl. Phys. 106, 113914 (2009).

[19] S.A. Baranov, V.N. Berzhanski, S.K. Zotov, V.L. Kokoz, V.S. Larin, A.V. Torcunov, Phys. Met. Metall. 67, 73 (1989).

[20] G.S.D. Beach, Nistor, C. Knutson, M. Tsoi, J.L. Erskine, Nat. Mater. 4, 741 (2005).

[21] L. V. Panina, H. Kato, K. Mohri, K. Kawashima, IEEE Trans. Magn. 29, 2524 (1993).

[22] R. Varga, K. Richter, A. Zhukov, V. Larin, IEEE Trans. Magn. 44, 3925 (2008).

[23] V. Zhukova, J. M. Blanco, V. Rodionova, M. Ipatov, A. Zhukov, J. Appl. Phys. 111, 07E311 (2012).

[24] A. Talaat, J. Alonso, V. Zhukova, E. Garaio, J.A. García, H. Srikanth, M.H. Phan, A. Zhukov, Sci. Rep. 6, 39300 (2016).

[25] H. Chiriac, T.A. Ovari, Gh. Pop, Phys Rev B 42 , 10104 (1995).

[26] A. Zhukov, J. Gonzalez, A. Torcunov, E. Pina, M.J. Prieto, A.F. Cobeńo, J.M. Blanco, V. Larin, S. Baranov, J. Magn. Magn. Mater. 203, 238 (1999).

[27] J. M. Blanco, V. Zhukova, M. Ipatov, A. Zhukov, Sensor Letters 11, 187 (2013).

[28] H. Chiriac, N. Lupu, G. Stoian, G. Ababei, S. Corodeanu, T.-A. Ovári, Crystals 7, 48 (2017).

[29] A. Zhukov, E. Kostitcyna, E. Shuvaeva, S. Kaloshkin, M. Churyukanova, V. Sudarchikova, A. Talaat, V. Zhukova, Intermetallics 44, 88 (2014).

[30] K.R. Pirota, L. Kraus, H. Chiriac, M. Knobel, J.Magn. Magn. Mater. 21, L243 (2000).

[31] A. Zhukov, V. Zhukova, J.M. Blanco, J. Gonzalez, J. Magn. Magn. Mater. 294, 182 (2005).

[32] A. Zhukov, A. Talaat, M. Ipatov, V. Zhukova, IEEE Magn. Lett. 6, 2500104 (2015).

[33] K. Narita, J. Yamasaki, H. Fukunaga, IEEE Trans. Magn. 16, 435 (1980).

[34] A. Zhukov, M. Churyukanova, S. Kaloshkin, V. Sudarchikova, S. Gudoshnikov,M. Ipatov, A. Talaat, J.M. Blanco, V. Zhukova, J. Electr. Mater. 45, 226 (2016).

[35] M. Churyukanova, V. Semenkova, S. Kaloshkin, E. Shuvaeva, S. Gudoshnikov, V. Zhukova, I. Shchetinin, A. Zhukov, Phys. Status Solidi A 213, 363 (2016).

[36] A. Zhukov, M. Vázquez, J. Velázqez, A. Hernando, V. Larin, J. Magn. Magn. Mater. 170, 323 (1997).

[37] N.A. Usov, A.S. Antonov, A.N. Lagar'kov, J. Magn. Magn. Mater. 185, 159 (1998).

[38] J.M. Blanco, A. Zhukov, J.Gonzalez, J. Phys. D:Appl. Phys. 32, 3140 (1999).

[39] G. Herzer, Amorphous, nanocrystalline soft magnets, in: Proceedings of the NATO Advanced Study Insititute on Magnetic Hysteresis in Novel Materials, Mykonos, Greece, 1-12 July 1996, Ed. G.C. Hadjipanayis, NATO ASI Series, Series E: Applied Sciences, Kluwer Academic Publishers, Dordrecht 1997, Vol. 338, p. 711.

[40] A. Zhukov, K Chichay, A. Talaat, V. Rodionova, J.M. Blanco, M. Ipatov, V. Zhukova, J. Magn. Magn. Mater. 383, 232 (2015). 
[41] A. Zhukov, A. Talaat, M. Ipatov, J.M. Blanco, V. Zhukova, J. Alloys Compounds 615, 610 (2014).

[42] A. Zhukov, M. Ipatov, M.Churyukanova, A. Talaat, J.M. Blanco, V. Zhukova, J. Alloys Compound. 727, 887 (2017).

[43] M.J. Garcia Prieto, E. Pina, A.P. Zhukov, V. Larin, P. Marin, M. Vázquez, A. Hernando, Sens. Actuators A 81/1-3, 227 (2000).

[44] V. Zhukova, J.M. Blanco, M. Ipatov, J. Gonzalez, M. Churyukanova, A. Zhukov, Scr. Mater. 142, 10 (2018).
[45] F.E. Luborsky, J.L. Walter, IEEE Trans. Magn. Mag-13, 953 (1977).

[46] J. Haimovich, T. Jagielinski, T. Egami, J. Appl. Phys. 57, 3581 (1985).

[47] Yu. Kabanov, A. Zhukov, V. Zhukova, J. Gonzalez, Appl. Phys. Lett. 87, 142507 (2005).

[48] A. Zhukov, A. Talaat, M. Churyukanova, S. Kaloshkin, V. Semenkova, M. Ipatov, J.M. Blanco, V. Zhukova, J. Alloys Compound. 664, 235 (2016). 